\title{
INTERNET METHODOLOGY - DIFFICULTIES AND OPPORTUNITIES OFFERED BY THE INTERNET IN CONDUCTING RESEARCH. ANALYSIS OF THE PHENOMENON IN THE LIGHT OF THE LITERATURE ON THE SUBJECT
}

\author{
KINGA FrącZKOWSKa \\ Institute of Pedagogy, Faculty of Social Sciences, \\ The Siedlce University of Natural Sciences and Humanities \\ ul. Żytnia 17/19, 08-110 Siedlce, Poland \\ e-mail address: kinga.fraczkowska@uph.edu.pl \\ ORCID: 0000-0003-0825-3654
}

\begin{abstract}
Aim. A theoretical review of the opportunities and functions of the application enabling the creation of internet surveys. Assessment of their use in accordance with own opinion and the analysis of the literature on the subject.

Methods. Analysis of the literature on the subject and a summary of the description of the survey conducted on the basis of a questionnaire examining the interesting phenomenon of e-methodology and an opinion on distance learning.

The authors analysed the advantages and disadvantages of the tools used in online research. The aforementioned experiences of the researchers refer to the experience gained during research on e-methodology.

Results and conclusion. The interest in this medium as a tool for social and psychological research has been growing rapidly in the last ten years. The very rapid development of technology that has taken place during the COVID-19 pandemic allowed us to easily conduct a research survey using the network, which can easily replace any labour-intensive data collection through inquiry. In addition, many different tools and techniques have been created that use the so-called "Global spider web" in conducting my own research. The researcher abandons direct contact with the respondent and devotes himself to the analysis of the phenomenon that interests him by analysing the given Internet environment, social groups that express themselves on a given topic or share their work on specific problems. However, the global network is not only a chance for good, innovative approach to research, but also threats and related difficulties. One of the most important weaknesses of the network is the fact that we can never be sure who is on the other end of the computer .

Cognitive value. Presentation of new ways of conducting scientific research via the network, presenting my own analysis of the advantages and disadvantages of the e-methodology phenomenon. Analysing the phenomenon of internet methodology, we learn that the tendency to participate in internet research is increasing. However, motivation to participate in internet research is not always sufficient, as mentioned by many researchers. There are many ways to influence the attractiveness of the research and the motivation to participate in the research. Thanks to this article, we also learn that it is
\end{abstract}


still necessary to conduct further methodological research on increasing the effectiveness of Internet research and minimising errors associated with it. The issues presented in this article reflect the current methodological knowledge. We can predict that as the Internet grows and new technical opportunities emerge - such as video-enabled applications, among others - new methodological issues will arise that may be of interest to researchers.

Keywords: Internet methodology, Internet, research, education

\section{INTERNET METHODOLOGY - WHAT IS THE PHENOMENON?}

The changed situation caused by the 2020 COVID-19 pandemic has been associated with a life change. Previous teachers, for example, worked at school with children who had direct contact with them. Currently, distance learning forces the student and the teacher to create relationships over the Internet. In many cases the Internet has become a constant social problem. How do we conduct surveys using anonymous surveys, if we cannot directly poll with them, study their behaviour? It turned out that scientists and researchers from around the world have failed in a serious way to conduct quantitative and qualitative research in the "global spider $w^{\prime \prime} b^{\prime}$. The techniques we use in online research are very similar to final tests identical to those we could use in the real world for respondents. It is also important that the network gives us the opportunity to conduct new, defined research, e.g. surveys (Kaczmarczyk, 2014). Moreover, respondents on the Internet feel more anonymous than when answering questions during an interview. This kind of advantage of the network undoubtedly encourages the respondents to participate in, for example, an online survey. By observing the current answer to the alternative question, alternative collaborative decision, alternative research version, online survey is the online survey. Why is online research so popular? There are many reasons, including that for providing it, we can quickly and directly check the responses of the respondents. One of the applications that quickly "won the hearts" of researchers, scientists, and even a student is Google Form. First, the program is very intuitive. With it, you can create a poll in several different ways (Batorski, 2004). It can be:

- a questionnaire with single-choice closed questions,

- a questionnaire with multiple-choice closed questions,

- survey with open questions,

- questionnaire with open and closed questions,

- a questionnaire with questions to be completed and a few that can be omitted while still meeting the specified conditions,

- survey with the use of the so-called Pop-ups, which are additional windows displayed on the respondent's answers (Żmijewska-Jędrzejczyk, 2004). 


\section{WHAT TOOLS ARE AVAILABLE FOR RESEARCHERS TO CONDUCT ONLINE SURVEYS AND GATHER OPINIONS IN THE VIRTUAL WORLD?}

By the end of 2020, it could be noticed that the market of tools for creating surveys and collecting responses from respondents looked very impressive. Using all the tools created by programmers around the world, not only could scientists collect the information they needed. Also, many companies more often began to evaluate their employees' work thanks to these types of tools. Moreover, employees of many companies could use them to check customer satisfaction with their offer. Additionally, they got to know their needs better, as well as their expectations regarding future transactions with their company. Therefore, it will be easier to collect ideas for your new, better product in the future and to improve the quality of running, for example, a company's website. All of these advantages also apply to online research. So what are the online research tools available for conducting opinion polls? (Kaniewska-Sęba, Leszczyński, \& Pilarczyk, 2006).

One of the applications is a Google Form. Thanks to it, we can not only create new documents, but also edit them and format the already created form. In addition, it is also a programme that facilitates the assessment of tests and surveys. The great advantage of the Google tool is combining it with the rest of the office suite, as well as the ability to quickly, even automatically export results to a Microsoft Excel spreadsheet. We can easily check the respondents' answers and their generated visualisations at every stage of the study in the form of: charts, tables comparing the statements of people participating in the on-line questionnaire. It is extremely helpful for the researcher himself, because he no longer has to spend as much time analysing the results as it was in the case of describing the data from the questionnaire carried out in the traditional form. The programme itself performs an initial analysis of the percentage of respondents' answers by creating tables and charts. The task of the researcher is only to describe them. The Google form also gives you the ability to change the theme and font, edit photos. In addition, you only need to copy the link to the form and we can easily send it to interested parties. The collection of the results takes place faster than during the implementation of the traditional survey, because the respondent only has to click on the "submit the form" button to get it to the recipient. Not only that, Google Form is also a free program that every owner of the Google browser has. Importantly, the researcher may also reserve the opportunity of recreating a given survey for a specific domain, thus having greater certainty that it will be personalised to his needs and will not be edited by unauthorised persons for this type of activity.

Another extremely popular tool is Survicate, which also offers surveys. They are easy to create, and also support multiple question options like a Google Form. The process of delivering the survey to the recipient is also similar. As in the above-mentioned tool, we can send our questionnaires by e-mail, display on the website or in the mobile application, all you need to 
do is copy the link and share it among the people you want to include in the survey. The only difference that appears in this tool is that it has a free and paid version and requires logging in via e-mail. The disproportion that can be noticed between these tools is also noticeable in the offer that Survicate presents. As described, application owners also provide the option of "embedding" the survey directly in their e-mail messages, which could contribute to a high completion rate of up to $83 \%$. Interestingly, they also propose the use of ready-made survey templates specific to the fulfillment of the goal specified by the client. These can be, for example, surveys checking customer satisfaction and their impressions. A questionnaire to check the usability of a given website. Thanks to this type of solution, it is easier for the researcher to assess whether the website created by him requires editing and in which direction it should go to meet the expectations of the recipient. It is a typical analytical tool that is easy to use. (Kociankowski, 2013).

Then I would like to present one of the more expensive tools that is primarily aimed at companies to research opinions on the website. The cost of creating an individual offer for those interested starts from $\$ 10,000$ per year. This may discourage a researcher from using that particular tool. In addition, it has limited opportunities to proactively conduct research and we can conclude that this may result in polarising respondents' opinions.

In addition, on the market we can find tools for researching opinions such as:

- mOpinion,

- Doorbell,

- Qualaroo,

- IdeaScale,

- Sugester,

- Crowdsignal,

- QuestionPro (Batorski, Olcoń-Kubicka, 2006).

All the programs listed above gather feedback freely. The recipient of the questionnaire decides whether he wants to complete it.

\section{QUANTITATIVE, QUALITATIVE AND EXPERIMENTAL RESEARCH ON THE WEB}

For many years scientists have been discussing whether there is a common method of empirical research that would become a universal solution for all fields of science. As we know well, at the moment there are many divisions of research methods and techniques. Methodological analysis of research brings us to a division in the humanities into at least two orientations: quantitative and qualitative. So what is quantitative research? Data collected in this type of research takes the form of sets or numbers that we can efficiently visualise using charts and tables. (Brzeziński, 2004). An exemplary fragment of a quantitative study is presented below. 


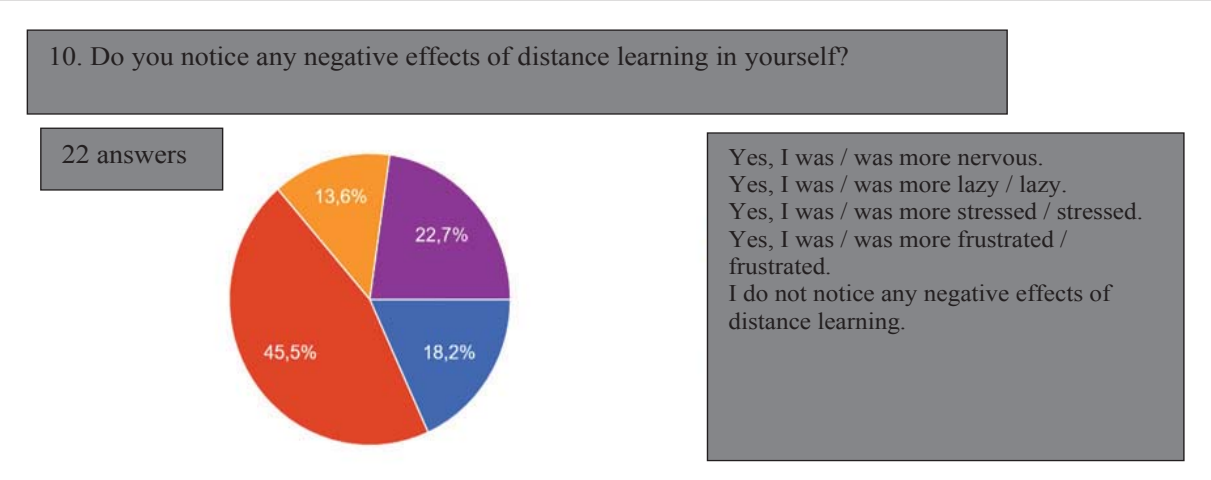

Fig. 1. Do you notice any negative effects of distance learning in yourself? - own research on the sense of security of students when remote learning is announced.

Analysing the above chart, we can see what percentage of the respondents answered the above question. Thanks to this type of solution, quantitative research plays a comparative role, which is conducive to making precise diagnoses of, for example, pedagogical situations at school. In addition, collecting several similar quantitative studies through the agreement of researchers may lead to a broader analysis of a given phenomenon and lead to a solution to the problem by applying appropriate preventive measures. So it is worth considering whether quantitative research can be carried out via the network? Of course, thanks to modern technology, we are able to carry out research of this type. In addition, it is worth noting that the subject of quantitative research, using an on-line survey, we can examine everything that can be counted (Maszke, 2008). Sometimes it is difficult for researchers to assess some phenomena or events because they cannot be measured as precisely as in the example above. These include, for example, difficult experiences and life experiences of people that they have experienced over many years. Qualitative, as opposed to quantitative, research does not take place by measurement or test, but by direct observation of the individual's behaviour in a given situation. So this type of research is hard to do with the web. Similarly, pedagogical experiments have little chance of being carried out remotely. Although some scientists use this type of research while working remotely (Frankfort-Nachmias, Nachmias, 2001).

\section{OPPORTUNITIES AND DIFFICULTIES POSED BY THE INTERNET IN CONDUCTING RESEARCH}

Thanks to the Internet, researchers have gained new opportunities to make their activities easier, much more convenient and cheaper. Thanks to them, scientists gain time, they do not have to set aside time for a meeting with the respondent to conduct, for example, a survey or an interview. One of the first, and also the most important, drivers of web-based research is the availability of a large 
number of subjects. It is especially valuable for quantitative research, as it enables us to reach a huge population not limited by geographic space. So it is much easier for us to research the opinion coming from abroad. It is enough to send a survey from your own home, which may be many kilometers away from people we want to ask for their opinions on the topic of interest to us. It plays an important role for synchronous research, because it enables obtaining information from many people who are located in different countries at the same time. We can also create a virtual conference room thanks to which we are able to conduct a group interview. Especially nowadays, it is a significant problem for researchers to reach people in difficult life situations, because the pandemic limits our opportunities to meet in a larger group. In addition, some studies require selecting a sample of people who have little time to meet and take part in the study. Also, the Internet copes well with these adversities, giving the respondent the freedom to set any time of the day to complete, for example, an online survey. Sometimes it is also necessary to reach certain subcultures, people who have the same interests. Sometimes the researcher wants to know the opinion on a specific topic of people with disabilities, single mothers staying at home with children or also seniors. Online research is also a great convenience for these social groups.

Another chance for research via the "global spider web" is to save the researcher time in analysing the results collected through, for example, a diagnostic survey. The data we obtain in online research is presented in digital form. Importantly, it is also ready for analysis. In a traditional interview, the researcher is forced to transcribe the respondents 'statements by handwriting, coding the respondents' answers. In an online interview, we can omit the issue of the written interview, because it happens automatically thanks to the recording of the entire conversation. As a result, we have a greater opportunity to undertake an immediate analysis of research questionnaire.

Unfortunately, the Internet, apart from the opportunities it offers, has several significant drawbacks and limitations. One of them is the frequent self-selection of respondents. Due to the fact that the sample consists mainly of volunteers who showed willingness to participate in the study. It is also important that any person can participate in online research any number of times. For the researcher, this means no verification of how many people actually took part in the survey and whether the remaining number does not apply to, for example, a few people who may have completed, , the survey repeatedly, for example. Therefore, it is correct to say that online research subjects cannot reflect a specific population, as we are not sure what the real representativeness of this research is.

According to Earl Babbie, the same criticism applies to telephone surveys, where the respondent may come from a completely different social group than the one he claims to be. Already in 1998, Joseph Hooton Taylor and George Terhanian tried to check the credibility of the results concerning the US elections: the government and the senate of the United States. To do this, they prepared and conducted as many as four Internet surveys. After summarising their activities, they came up with surprising results. It turned out that 21 out of 22 winners of the elections were correctly selected, which accounted for $95 \%$ of the actual results. In 
addition, the results collected from online surveys differed from the real results by an average of $6.8 \%$. Interestingly, when comparing them with the telephone polls, they noticed that they differed from the results obtained after the elections by $6.2 \%$ on average. Another difficulty that we can notice in the online survey is the adaptation of research techniques to the new realities. Many people with no experience in the field of creating research do not realise what a complex and very demanding process the research preparation stage is. They think that in order to create research, all you need is a good idea and approximately 15 minutes of time to construct an appropriate research tool. However, the truth is quite different. Above all, we should have adequate and well-established knowledge of research methodology, e.g. pedagogical, if we want to include a specific group of students in the research. Then we can designate the subject, research goal, technique and research method, and only then are we ready to create the correct research tool. Of course, even in 10 minutes you can prepare such a study, but the quality and reliability of the results that you obtain may be subject to discussion. Poorly structured questions in the questionnaire may result in, for example, obtaining incorrect, unreliable results. It is also worth paying attention to the fact that when conducting online research, we do not have the opportunity to acquire, for example, respondents' comments about the research, which often provide very important feedback on the research tool or the topic covered in it (Babbie, 2004).

\section{Conclusion}

Undoubtedly, the Internet is a medium that allows us to collect responses and data from the social groups we are interested in in a modern, different way. We can analyse them quickly and register and save human statements. We also owe the network the ability to evaluate a website, electronic messages (e-mails), statements on discussion forums or in internet diaries, which are called blogs. Our daily social life during the COVID-19 pandemic has largely shifted to this medium. Therefore, scientists who want to "follow the spirit of the times" find it much more difficult to remain indifferent to the Internet and the phenomena that can be noticed in it. Online conference room conversations, which are automatically recorded, are a good and easy-to-perform alternative to costly and time-consuming face-to-face interviews with the recipient. On the other hand, online questionnaires become a substitute for questionnaires handed out directly to the person taking part in the study. Thus, the "global network" becomes, in a way, a great place for scientists to explore. It should also be remembered that the Internet methodology and every area of human life have advantages and disadvantages. The researcher's task is therefore to personally assess whether conducting online research brings more benefits, opportunities than losses and difficulties for him. Sometimes the choice of even the most modern solutions, ie collecting data via the network, does not guarantee that our "broker" will be flawless. Sometimes a change in the method of data collection may turn the disadvantages of a given method into advantages and vice versa. 


\section{REFERENCES}

[1] Babbie E., (2004). Social research in practice. Warsaw: PWN.

[2] Batorski D., (2004). Towards the information society. In: Czapiński J. and Panek T., Social diagnosis: Conditions and quality of life of Poles (pp. 195-235). Warsaw: University of Finance and Management.

[3] Batorski D., (2005). Social aspects of new technologies. In: J. Czapiński and T. Panek, Social diagnosis 2005: Conditions and quality of life of Poles (pp. 27-28 and 214-231). Warsaw: University of Finance and Management.

[4] Batorski D., Olcon-Kubicka M., (2006). Conducting research via the Internet - basic methodological issues, Sociological Studies, 2, 106-108.

[5] Brzeziński J., (2004). Methodology of psychological research. Warsaw: PWN.

[6] Frankfort-Nachmias Ch., Nachmias D., (2001). Research methods in sciences, Poznań: Zysk i S-ka.

[7] Kaczmarczyk S., (2014). Marketing research. Methodological basics. Warsaw: PWE.

[8] Kaniewska-Sęba A., Leszczyński, G., Pilarczyk, B. (2006). Marketing research on the business-to-business market. Krakow: Economic Printing House.

[9] Kociankowski M., (2013). The typology of research is becoming more and more comprehensible. Marketing Research, 14, 191-192.

[10] Maszke A. W., (2008). Methods and techniques of pedagogical research. Rzeszow: Publishing House of the University of Rzeszów.

[11] Żmijewska-Jędrzejczyk T., (2004). Internet research. In: P. B. Sztabiński, F. Sztabiński and Z. Sawiński, New methods, new initiatives in social sciences (pp. 241-259). Warsaw: IFiS PAN Publishing House. 